\title{
The association of cardiovascular autonomic dysfunction and the prediction of COPD can be explained by neurohumoral activation
}

To the Editor:

I read with great interest the recent work by RICCI et al. [1] published in the European Respiratory Journal. This insightful population-based study followed a very large cohort without baseline airflow obstruction for 32 years. Interestingly, baseline orthostatic blood pressure decrease, as well as increased resting heart rate, was associated with reduced lung function during follow-up. The authors consequently suggested that subtle signs of cardiovascular autonomic dysfunction may precede the development of chronic obstructive pulmonary disease (COPD).

I would like to take this notion further and suggest that the observed association might be explained by subtle neurohumoral activation. With a variety of methods, we and others have described striking neurohumoral activation in patients with moderate to severe COPD [2-5]. Several mechanisms, such as hypoxia, chemoreflexes, impaired baroreflexes, less physical activity and increased work of breathing, are likely to contribute to sympathetic activation in COPD.

Since microneurography as a gold standard for the evaluation of sympathetic activity is a challenging method and has never been performed in a population-based cohort, it is not possible to prove this assumption. However, in other diseases, such as heart failure and obstructive sleep apnoea, the concept of neurohumoral activation and autonomic nervous system imbalance with increased sympathetic activation and reduced parasympathetic activity has long been recognised, and is related to increased resting heart rate, as well as increased morbidity and mortality $[6,7]$.

Thus, it is reasonable to suggest that orthostatic blood pressure decrease and increased resting heart rate, as observed by RICCI et al. [1], are a sign of subtle neurohumoral activation. This neurohumoral activation might indeed contribute to progression of lung disease by pro-inflammatory activity, as RicCI et al. [1] suggested in their discussion.

Whatever the underlying mechanism, the novel findings by Ricci et al. [1] shed light on the importance of the autonomic nervous system in the pathogenesis of chronic diseases such as COPD.

@ERSpublications

Clinical markers of subtle cardiovascular autonomic dysfunction predict incident COPD: the potential role of neurohumoral activation http://ow.ly/mUcW30jMnPC

Cite this article as: Andreas S. The association of cardiovascular autonomic dysfunction and the prediction of COPD can be explained by neurohumoral activation. Eur Respir J 2018; 51: 1800737 [https:// doi.org/10.1183/13993003.00737-2018].

Stefan Andreas $\oplus^{1,2}$

${ }^{1}$ Lungenfachklinik Immenhausen, Immenhausen, Germany. ${ }^{2}$ Kardiologie und Pneumologie, Universitätsmedizin Göttingen, Göttingen, Germany.

Correspondence: Stefan Andreas, Lungenfachklinik Immenhausen, R. Koch Str. 3, Immenhausen 34376, Germany. E-mail: Stefan.andreas@med.uni-goettingen.de

Received: April 182018 | Accepted: April 192018

Conflict of interest: None declared. 


\section{References}

1 Ricci F, Wollmer P, Engström G, et al. Markers of cardiovascular autonomic dysfunction predict COPD in middle-aged subjects. Eur Respir J 2018; 51: 1702481.

2 Andreas S, Anker SD, Scanlon PD, et al. Neurohumoral activation as a link to systemic manifestation of chronic lung disease. Chest 2005; 128: 3618-3624.

3 Mohammed J, Meeus M, Derom E, et al. Evidence for autonomic function and its influencing factors in subjects with COPD: a systematic review. Respir Care 2015; 60: 1841-1851.

4 Luthje L, Raupach T, Michels $\mathrm{H}$, et al. Exercise intolerance and systemic manifestations of pulmonary emphysema in a mouse model. Respir Res 2009; 10: 7 .

5 Heindl S, Lehnert M, Criée CP, et al. Marked sympathetic activation in patients with chronic respiratory failure. Am J Respir Crit Care Med 2001; 164: 597-601.

6 Floras JS. Clinical aspects of sympathetic activation and parasympathetic withdrawal in heart failure. J Am Coll Cardiol 1993; 22: Suppl. A, 72a-84a.

7 Andreas S, Eichele G. Sleep apnoea: time to consider clock genes. Eur Respir J 2008; 32: 1-2.

Copyright (CERS 2018

\section{Dysregulation of the "inflammatory reflex" with abnormal neurohumoral activation may contribute to proinflammatory activity driving the progression of COPD}

From the authors:

We would like to thank S. Andreas for his interest in our study [1] and for the interesting comments. We agree with the insightful reasoning that subtle neurohormonal activation may be one of the explanations underlying the longitudinal association between subtle signs of cardiovascular autonomic dysfunction and higher risk of chronic obstructive pulmonary disease (COPD). In parallel to the findings in heart failure [2] and COPD [3], manifest clinical entities of cardiovascular autonomic dysfunction, such as orthostatic hypotension [4,5] and postural orthostatic tachycardia syndrome [6], are all associated with clear signs of neurohormonal activation and inflammatory activation playing in the background.

The discovery that cholinergic neurons inhibit acute inflammation gave birth to the concept of "inflammatory reflex", a "hard-wired" connection between the nervous and immune systems, where the ascending link between the nucleus tractus solitaries and the paraventricular nucleus provides a pathway that can modulate both vagus efferent activity inhibiting cytokine synthesis and neuro-hormonal anti-inflammatory responses $[7,8]$. One hypothesis is that autonomic nervous system imbalance, with increased sympathetic activation and reduced parasympathetic activity, might be the inception of a dysregulation of the inflammatory reflex with abnormal neurohumoral activation contributing to local and systemic pro-inflammatory activity driving the progression of COPD.

Further investigations should explore the complex relationship between cardiovascular autonomic dysfunction and neurohormonal activation at the crossroad between inflammation and cardiovascular and pulmonary disease. Ultimately, this effort might identify specific neurohormonal mediators and

@ERSpublications

Dysregulation of the "inflammatory reflex" with abnormal neurohumoral activation may contribute to proinflammatory activity driving the progression of COPD http://ow.ly/e9xx30k4kCk

Cite this article as: Ricci F, Wollmer P, Engström G, et al. Dysregulation of the "inflammatory reflex" with abnormal neurohumoral activation may contribute to proinflammatory activity driving the progression of COPD. Eur Respir J 2018; 51: 1800806 [https://doi.org/10.1183/13993003.00806-2018]. 
pathophysiological mechanisms involved in the development and progression of COPD, which can be targeted in preventive or therapeutic efforts.

Fabrizio Ricci ${ }^{1,2}$, Per Wollmer ${ }^{3,4}$, Gunnar Engström ${ }^{1}$, Artur Fedorowski ${ }^{1,5}$ and Viktor Hamrefors ${ }^{1,6}$

${ }^{1}$ Dept of Clinical Sciences, Lund University, Malmö, Sweden. ${ }^{2}$ Institute for Advanced Biomedical Technologies, Dept of Neuroscience, Imaging and Clinical Sciences, "G.d'Annunzio" University, Chieti, Italy. ${ }^{3}$ Dept of Translational Medicine, Lund University, Malmö, Sweden. ${ }^{4}$ Dept of Medical Imaging and Physiology, Skåne University Hospital, Malmö, Sweden. ${ }^{5}$ Dept of Cardiology, Skåne University Hospital, Malmö, Sweden. ${ }^{6}$ Dept of Internal Medicine, Skåne University Hospital, Malmö, Sweden.

Correspondence: Viktor Hamrefors, Clinical Research Centre, Lund University, Jan Waldenströms Gata 35, Building 91, Floor 12, SE-205 02 Malmö, Sweden. E-mail: Viktor.Hamrefors@med.lu.se

Received: April 292018 | Accepted: May 012018

Conflict of interest: None declared.

\section{References}

1 Ricci F, Wollmer P, Engstrom G, et al. Markers of cardiovascular autonomic dysfunction predict COPD in middle-aged subjects. Eur Respir J 2018; 51: 1702481.

2 Floras JS. Clinical aspects of sympathetic activation and parasympathetic withdrawal in heart failure. J Am Coll Cardiol 1993; 22: 72A-84A.

3 Andreas S, Anker SD, Scanlon PD, et al. Neurohumoral activation as a link to systemic manifestations of chronic lung disease. Chest 2005; 128: 3618-3624.

4 Johansson M, Ricci F, Aung N, et al. Inflammatory biomarker profiling in classical orthostatic hypotension: Insights from the SYSTEMA cohort. Int J Cardiol 2018; 259: 192-197.

5 Johansson M, Ricci F, Aung N, et al. Proteomic profiling for cardiovascular biomarker discovery in orthostatic hypotension. Hypertension 2018; 71: 465-472.

6 Hamrefors V, Spahic JM, Nilsson D, et al. Syndromes of orthostatic intolerance and syncope in young adults. Open Heart 2017; 4: e000585.

7 Tracey KJ. The inflammatory reflex. Nature 2002; 420: 853-859.

8 Pavlov VA, Wang $\mathrm{H}$, Czura CJ, et al. The cholinergic anti-inflammatory pathway: a missing link in neuroimmunomodulation. Mol Med 2003; 9: 125-134. 